\title{
Kultur der türkischen Diaspora in Deutschland und ihre Implementierung im Germanistikstudium. Ein praxisorientierter Blick aus Polen
}

\section{Culture of the Turkish Diaspora in Germany and its Implementation in German Studies. A Practice-Oriented View from Poland}

Krzysztof OKOŃSKi' [

'Prof. Dr., Uniwersytet Kazimierza Wielkiego w Bydgoszczy, Instytut Nauk o Kulturze, Bydgoszcz, Poland

ORCID: K.O. 0000-0002-8779-2535

\section{Corresponding author:} Krzysztof OKOŃSKI

Uniwersytet Kazimierza Wielkiego w Bydgoszczy, Instytut Nauk o Kulturze, Bydgoszcz, Poland

E-mail: k.okonski@ukw.edu.pl

Submitted: 11.05.2020

Revision Requested: 14.09.2020

Last Revision Received: 02.10.2020

Accepted: 23.10 .2020

Citation: Okoński, K. (2020). Kultur der türkischen Diaspora in Deutschland und ihre Implementierung im

Germanistikstudium. Ein praxisorientierter Blick aus Polen. Alman Dili ve Edebiyatı Dergisi - Studien zur deutschen Sprache und Literatur, 44, 69-90.

https://doi.org/10.26650/sdsl2020-0012

\begin{abstract}
DEUTSCH)
Die Kultur der türkischen Diaspora wird immer häufiger zum Forschungsgegenstand der Germanistik in Deutschland und im Ausland. Seit etwa zehn Jahren steigt die Zahl polnischer Germanisten, die sich mit diesem Themenkomplex wissenschaftlich und didaktisch beschäftigen. In dem vorliegenden Beitrag wird der Versuch unternommen, Möglichkeiten und Probleme bei der Implementierung der deutschtürkischen Kultur in der germanistischen Lehrpraxis in Polen an ausgewählten Beispielen zu präsentieren. Der Artikel bietet einen Überblick über kulturelle und historische Aspekte der polnisch-türkischen Beziehungen, die die Einbeziehung der deutschtürkischen Kultur im Germanistikstudium in Polen fördern können. Weil der Einfluss deutscher Künstler türkischer Herkunft auf die Entwicklung der Kultur in Deutschland immer größer wird, ist es auch notwendig, eine Frage nach ihrer Rezeption und Präsenz in Polen zu stellen. Im analytischen Teil des vorliegenden Textes wurden 24 Diplomarbeiten an der Kazimierz-WielkiUniversität Bydgoszcz/Polen in thematischen Gruppen zusammengestellt und besprochen. Um sich einen Einblick in die Erwartungen und Kompetenzen künftiger Germanisten verschaffen zu können, wurden die Studierenden in einer Umfrage nach ihrer Stellungnahme zu einer eventuellen Einbeziehung dieser Problematik im Germanistikstudium gefragt. Die Datenerhebungen wurden 2007 und 2020 durchgeführt. Dadurch ist es möglich festzustellen, inwieweit die in letzter Zeit veränderten politischen und gesellschaftlichen Bedingungen (Migrationskrise, Nationalismus, eine sichtbare Präsenz türkischer Studenten an polnischen Universitäten) die interkulturelle Öffnung polnischer Studierenden (am Beispiel der Germanistik an der Kazimierz-Wielki-Universität) beeinflussen.
\end{abstract}

Schlüsselwörter: Migration, Deutschtürken, Polen, Didaktik, Rechtsruck 


\begin{abstract}
ENGLISH)
The culture of the Turkish diaspora is increasingly becoming an object of research in German studies in Germany and abroad. The number of German studies experts in Poland who analyze this topic from the academic and research perspective has been increasing over the last ten years. This article attempts to present possibilities and problems in the implementation of the German-Turkish culture in academic didactic contexts (i.e., studies on German language and literature) in Poland on selected examples. This article summarizes the cultural and historical aspects of Polish-Turkish relations that can facilitate the inclusion of the German-Turkish culture in German studies in Poland. Due to the steady increase in the influence of German artists of Turkish origin over the development of culture in Germany, discussing their reception and presence in Poland is also necessary. In the analytical section of this paper, 24 diploma theses of students of German studies at Kazimierz Wielki University in Bydgoszcz, Poland, were collected and discussed in subject-related fields. To gain insights into the expectations and competencies of the students, they were surveyed for their opinion on the potential inclusion of the German-Turkish culture in German studies. Data collection was conducted in 2007 and 2020. This data enabled us to determine the extent at which recently changed political circumstances (e.g., migration crisis, xenophobia, growing nationalism, presence of Turkish students at Polish universities) affected the students' willingness to embrace and accept an "ethnic paradigm shift" in the curriculum of German studies.

Keywords: Migration, German-Turks, Poles, didactics, shift to the right
\end{abstract}

\title{
EXTENDED ABSTRACT
}

The culture of the Turkish diaspora is increasingly becoming an object of research in German studies in Germany and abroad. The number of German studies experts in Poland who deal with this topic from the academic and research perspective has been increasing over several years. Intercultural research in Polish and German studies has traditionally included topics such as German-Polish cultural transfer in the past and present, interference of the German language with the Polish language, or images of Poles and Germans in literature or films. Being neighbors for 1,000 years, and the fact that several German writers and artists (e.g., Günter Grass, Janosch, Horst Bienek or Klaus Kinski) have been born in the German-Polish border area or in former German provinces (e.g., Silesia, Danzig, Zoppot) are reasons why focus on this research was more relevant to the Polish experts on German studies than research on the culture of Turkish migrants in Germany. Moreover, after 1989, a need to conduct more research on topics that had been taboo before that period emerged (e.g., the anti-German propaganda in the Communist era). The development of the German-Turkish cultural elite entails new research premises along with the need to incorporate topics related to migrant culture into the academia. This article attempts to present possibilities and problems in the implementation of the German-Turkish culture in academic didactic contexts (e.g., studies on German language and literature) in Poland using select examples. In this context, the outcome of Polish students' intercultural motivation in German studies is more of a pragmatic competence than a cultural competence because they acquire additional skills and competencies 
enabling them to work in international environments (e.g., transnational teams, global companies). This article summarizes the cultural and historical aspects of Polish-Turkish relations that can facilitate the inclusion of the German-Turkish culture in German studies in Poland. Due to the influence of German artists of Turkish origin over the development of culture in Germany steadily increasing, discussing their reception and presence in Poland is also necessary. Although the literature of Turkish writers, especially the creative output of Orhan Pamuk and Elif Şafak, has often been reviewed, published, and broadcast as radio plays by Polskie Radio in Poland, the number of novels by German-Turkish authors remains limited.

Paraschos Berberoğlu, in 1995, said that the intercultural perspective of teaching German as a foreign language requires teachers to acquire extra skills, such as an open and creative attitude, knowledge of the diverse cultures represented in school communities, or applying adequate teaching and learning aids. In the analytical section of this paper, 24 diploma theses of students of German studies at Kazimierz Wielki University in Bydgoszcz, Poland, were collected and discussed in subject-related fields. To gain insights into the expectations and competencies of the students, they were surveyed for their opinion on the potential inclusion of the German-Turkish culture in German studies. Data collection was conducted in 2007 and 2020. The relationship between Poles and migrants was stable until 2014 and 2015. Unlike Germany, the right-wing radicalism in Poland was not directed against immigrants or Muslims because at that time, almost no immigration from Muslim countries, including Turkey, occurred. A nationalist change of the social climate in Poland after 2015 was a reaction to ISIS terrorist attacks. This stability changed because of mass migrations from Ukraine to Poland and a visible presence of Turkish students, and-more rarely-migrant workers from Turkey. This phenomenon enables us to determine the extent at which recently changed political circumstances (e.g., migration crisis, xenophobia, growing nationalism, presence of Turkish students at Polish universities) affected the students' willingness to embrace and accept an "ethnic paradigm shift" in the curriculum of German studies. 


\section{Ziel, Methoden und Forschungsgegenstand}

Der vorliegende Beitrag stellt den Versuch dar, didaktische Potenziale der deutschtürkischen Kultur und ihre praktische Umsetzung im Germanistikstudium in Polen zu beleuchten. Der Aufbau des Artikels resultiert aus der Notwendigkeit, den gesellschaftlichen und kulturhistorischen Hintergrund zu präsentieren, um die Bedingungen für die Beschäftigung mit dieser Thematik an einer polnischen Universität auszuloten. Der interkulturelle Ansatz in der germanistischen Forschung hat in Polen zum Teil andere Traditionen als in Deutschland und wurde nach 1989 nicht nur durch unterschiedliche Facetten der Nachbarschaft, sondern auch durch die Bestrebung geprägt, an die bisher u.a. wegen der Zensur verdrängten Themen in diesem Bereich anzuknüpfen. Der Artikel umfasst außerdem Aspekte polnischer Migrationsdiskurse im Blick auf den sich seit 2015 abzeichnenden Rechtsruck. Im analytischen Teil des vorliegenden Aufsatzes wurden zunächst 24 Diplomarbeiten in thematischen Gruppen zusammengestellt und besprochen, um das Interesse der Studierenden an der deutschtürkischen Kultur und an deren didaktischen Umsetzung aufzuzeigen. Als nächstes wurden die Ergebnisse einer empirischen Studie über die Möglichkeiten, ausgewählte Aspekte der Migrantenkultur in die akademische Lehrpraxis zu integrieren, präsentiert. Sie wurde in zwei Datenerhebungen (2007 und 2020) durchgeführt.

\section{Einleitung}

Die Erforschung des Kulturtransfers zwischen Polen und Deutschland hat in der polnischen Germanistik eine lange Tradition und bleibt nach wie vor eine wichtige Prämisse in der wissenschaftlichen und didaktischen Praxis, nicht nur vor dem Hintergrund einer wechselhaften Geschichte der bilateralen Beziehungen, sondern auch angesichts der Gegenwart. Die Relevanz dieser Thematik lässt sich ebenfalls mit ihrer praktischen Umsetzung belegen - Deutschland ist der größte Handelspartner Polens. Für die Bundesrepublik ist Polen sechswichtigster Handelspartner, auf der Liste der Herkunftsländer der Ausländer in Deutschland bleibt Polen auf dem zweiten Platz hinter der Türkei, Deutsch ist nach Englisch die zweitbeliebteste Fremdsprache in Polen und das Bild der Deutschen zählt zu den häufigsten ethnischen Motiven in der polnischen Kultur.

Im Blick auf die vorhin genannten Tatsachen scheint die Beschäftigung mit historischen und gegenwärtigen Aspekten der Beziehungen zwischen beiden Ländern eine groß angelegte Aufgabe zu sein und zwar nicht nur wegen der politischen 
Umstände, unter denen sich die polnische Germanistik vor 1989 entwickelte und erst nach dem Systemwechsel z.B. an übernationale Traditionen dieser Disziplin in Polen anknüpfen durfte (Kunicki \& Zybura, 2011). Ein Blick auf die Landkarte Polens verdeutlicht, dass viele berühmte deutsche Autoren (u.a. Horst Bienek, Janosch, Günter Grass oder Marcel Reich-Ranicki) im deutsch-polnischen Grenzgebiet zur Welt kamen.

Im Dialog mit der interkulturellen Germanistik (Nayhauss \& Kuczyński, 1993) heißt der Band von 1993, den - bestimmt nicht zufällig - die Grafik Unterwegs in Polen von Günter Grass eröffnet. Er enthält Beiträge u.a. über solche Themen, wie Joseph Eichendorff und Schlesien, Gerhart Hauptmann (ebenfalls ein Schlesier), das deutsch-polnische Verhältnis im 20. Jahrhundert als sozial-kulturelles Phänomen, Fremdes und Eigenes in der deutschsprachigen Literatur, die Erfahrung des Heimatverlusts oder das Gedicht Polnische Fahne des erwähnten Danziger Grass. Obwohl man diese Auswahl als beschränkt erachten kann, steht sie exemplarisch für ein breites und für die polnische Germanistik relevantes Forschungsfeld.

Die vorhin erwähnte Konzentration der Germanistik in Polen auf die polnische Komponente der Multikulturalität in Deutschland ist vor dem historischen, kulturellen und geographischen Hintergrund verständlich. Die Bildung einer Kulturelite, die aus der ökonomisch geprägten Einwanderung in die BRD hervorgegangen ist, bedeutet jedoch neue Forschungsprämissen und nicht zuletzt auch die Notwendigkeit, Inhalte aus einem anderen Kulturkreis in die didaktische Praxis einzubeziehen. Die Sensibilisierung der Studenten für solche Begriffe, wie hybride Identitäten, Leitkultur, Assimilation, Kiezdeutsch, oder Frauenrechte ist zwar notwendig, um sich mit den von deutschtürkischen Künstlern dargestellten Problemen auseinanderzusetzen, sie findet dennoch sehr selten Zugang zur germanistischen Forschung und Lehre in Polen. Bei einer genaueren Betrachtung aus der polnischen Perspektive stellt sich heraus, dass Auswanderung, Patriarchat, häusliche Gewalt oder Identitätsfragen gleichermaßen den Diskurs in der Türkei und in Polen prägen: Die vermeintlich exotischen Probleme kommen überraschend bekannt vor.

\section{Wahrnehmung der (deutsch)türkischen Kultur in Polen}

Eine relativ späte Annäherung der polnischen Germanistik an den Themenkomplex ,Türkische Migration nach Deutschland' lässt sich auch mit der politischen Entwicklung erklären. Nach dem Zusammenbruch des Kommunismus eröffneten sich neue 
Perspektiven für die Erforschung von Themen, die vor 1989 z.B. wegen der Zensur nicht behandelt werden durften (polnische Exilkultur, DDR-kritische Literatur, antideutsche Prägung der Propaganda usw.). Hinzu kam, dass die Volksrepublik Polen als ein ethnisch homogenes Land wahrgenommen werden wollte. Die Existenz von nationalen Minderheiten, besonders der deutschen Minderheit, wurde in der kommunistischen Zeit geleugnet bzw. marginalisiert. Probleme, die für eine multikulturelle Gesellschaft typisch waren, konnten deshalb sowohl den Machthabern als auch vielen Bürgern als fremd erscheinen. Das Buch Ganz unten von Günter Wallraff, das in Polen unter dem Titel Na samym dnie 1988 erschien, verhalf dem Einwanderungsland Deutschland seine Türken zu erblicken. Angesichts der katastrophalen wirtschaftlichen Lage Polens Ende der achtziger Jahre und der verbreiteten Hoffnungslosigkeit stellte ein solches Buch (wider Willen des Autors) ein gewisses propagandistisches Potenzial dar, weil es sich dafür eignete, die prekären Arbeitsverhältnisse in der ,bösen' kapitalistischen Bundesrepublik zu überhöhen. Die Notwendigkeit, andere Themen nach 40 Jahren Unfreiheit nachzuholen, trug ebenfalls dazu bei, dass die Problematik der türkischen Migration in den Hintergrund trat.

Es ist jedoch anzumerken, dass das Wissen um wechselhafte türkisch-polnische Verhältnisse (beide Länder hatten einst eine gemeinsame Grenze) weder in der VR Polen, noch in der III Republik (nach 1990) verdrängt wurde. Es lässt sich mit zahlreichen Beispielen belegen, die man sogar jedem Schulkanon entnehmen kann: Kriege zwischen dem Königreich Polen und dem Osmanischen Reich mit ihren späteren Folgen (Bömelburg, Rohdewald \& Uffelmann, 2016), die entscheidende Rolle der Polen im Kampf gegen die türkische Belagerung Wiens 1683, die bis heute von einer besonderer Bedeutung für das historische Gedächtnis der Polen ist, Tod des polnischen Nationaldichters Adam Mickiewicz in Istanbul (1855), Verdienste des Generals Józef Bem/ Murad Paşa, der Held Polens, Ungarns und der Türkei war oder die Gründung des polnischen Dorfes Adampol/ Polonezköy durch ehemalige polnische Aufständische, die vom Osmanischen Reich aufgenommen wurden. Nicht zuletzt ist auch zu erwähnen, dass das Osmanische Reich die Teilungen Polens durch Preußen, Österreich und Russland nie anerkannt hat. Ein Versuch, in polnischen Schulbüchern nach Informationen über die Gründung der Türkischen Republik, über den Unabhängigkeitskrieg oder über die von Mustafa Kemal Paşa eingeleiteten Modernisierungsprozesse zu suchen, ist jedoch vergeblich. 
Die Eingliederung Polens in den sowjetischen Machtbereich und die Bindung der Türkei an den Westen wirkten sich nicht nur auf den politischen Dialog, sondern auch auf den Kulturaustausch aus. Als der türkische Vorzeigedichter wurde in der Volksrepublik Polen Nâzım Hikmet gefeiert, der sich nicht nur zu seiner marxistischen Weltanschauung, sondern auch zu polnischen Vorfahren öffentlich bekannte. Witold Szabłowski widmete ihm ein Kapitel in seiner populären Reportagensammlung aus der Türkei (Szabłowski, 2018): „Fühlte er sich damals ein wenig ein Pole zu sein? 1958 sagte er bei einer Lesung im Club der Internationalen Presse und Literatur: „Ich habe eine polnische Nase, Haare, die hell waren, bevor sie grau geworden sind und viel Romantik - wohl polnischer Herkunft" (Szabłowski, 2018, S. 40). Ein Jahr später fügte er in einem Interview für die Zeitschrift „Orka“ hinzu: „Von den Ländern, die mir zur Wahl standen, wählte ich das Land meines Ururgroßvaters" (Szabłowski, 2018, S. 40) - gemeint war der polnische Aufständische und türkische General Konstanty Borzęcki/Mustafa Celâleddin Paşa.

Seitdem ist es um die türkische Literatur in Polen still geworden: „Als Turkologin kann ich nur bereuen, dass die türkische Literatur seit vielen Jahren nicht mehr ins Polnische übersetzt wird“ (Ayşen Kaim, 2015) - stellte 2014 die Warschauer Literaturwissenschaftlerin und Übersetzerin Agnieszka Ayşen Kaim in einem Interview für den staatlichen Radiosender Program I Polskie Radio fest In diesem Gespräch, das anlässlich des Türkischen Tages im Polnischen Rundfunk geführt wurde, erklärte sie, wie es in Polen um die türkische Literatur in Polen bestellt sei. Die auch hierzulande viel kommentierte Verleihung des Nobelpreises an Orhan Pamuk und enthusiastische Reaktionen der polnischen Literaturkritik bilden dennoch nur einen bescheidenen Hintergrund für spektakuläre Erfolge der türkischen Fernsehserie Muhteşem Yüzyıl/Wspaniałe stulecie. Agnieszka Aysen Kaim äußerte sich dazu wie folgt:

2014 haben wir den 600. Jahrestag der polnisch-türkischen diplomatischen Beziehungen gefeiert. Es stellt sich jedoch heraus, dass die aus diesem Anlass organisierten Galaabende und Events die Wahrnehmung der Türkei durch die Polen weniger als die Ausstrahlung einer Fernsehserie über einen Herrscher aus dem 16. Jahrhundert beeinflusst haben. (Ayşen Kaim, 2015)

Obwohl türkische Künstler, wie Orhan Pamuk, Elif Şafak oder Semih Kaplanoğlu für ihre literarischen oder filmischen Werke von polnischen Kritikern meistens mit sehr guten Rezensionen belohnt werden, begeistert sich das Fernsehpublikum in Polen vor allem für weniger anspruchsvolle Titel: Einem episodenhaften Sturm des Sängers Tarkan auf 
polnische Charts (Şımarık, 1997) folgten im letzten Jahrzehnt Erfolge solcher TV-Serien, wie Binbir Gece/Tysiąc i jedna noc, Kiraz Mevsimi/Sezon na miłość, Aşk-ı Memnu/Zakazana miłość oder Fatmagül'ün Suçu Ne?/Grzech Fatmagül.

Während die Literatur türkischer Autoren (besonders das Schaffen von Orhan Pamuk und Elif Şafak) nicht nur als Hörspiele des Senders Polskie Radio erscheint, sondern auch rezensiert und von Buchverlagen herausgegeben wird (13 polnische Übersetzungen der Romane und Erzählungen von Pamuk zwischen 2006 und 2017), ist der Zugang zum Werk deutschtürkischer Schriftsteller auf einige Bücher beschränkt: Leyla (Feridun Zaimoglu), Bittersüße Heimat. Bericht aus dem Inneren der Türkei/Słodko-gorzka ojczyzna. Raport z serca Turcji (Necla Kelek), Die Brücke vom Goldenen Horn/Most nad Złotym Rogiem (Emine Sevgi Özdamar) und Felidae - Das Duell, Salve Roma!/Pojedynek, Salve Roma! (Akif Pirinçci). Als einen Grund für diese begrenzte und nicht immer repräsentative Wahl kann man u.a. Schwierigkeiten in der Übersetzung ins Polnische des deutschtürkischen Etnolekts nennen (Jonczyk, 2014). Neben literaturwissenschaftlichen Texten einiger polnischer Germanisteninnen oder Artikeln in der Presse, sind Lesungen an Universitäten (Necla Kelek, Katholische Universität Lublin, 2008), in Filialen des Goethe-Instituts, in Buchhandlungen (Feridun Zaimoglu in Kraków/Krakau, Warszawa/Warschau und Poznań/ Posen, 2008) oder bei Literaturfestivals (Yadé Kara und Necla Kelek, Wrocław/Breslau, 2018) die einzige Möglichkeit, diese Literatur kennenzulernen.

\section{Verhältnisse zwischen Polen und Türken in den Zeiten des Rechtsrucks in Polen}

Bei der Wahl ihrer Lektüren oder Filme richten sich polnische Leser selbstverständlich nicht nur nach ästhetischen und künstlerischen Kriterien. So wie der Umbruch von 1989 den Zugang zur verbotenen Literatur eröffnet hat, hat auch die Integration Polens mit dem Westen, insbesondere mit der EU indirekte Folgen für kulturelle Vorlieben der Polen. Diese Öffnung auf Westeuropa bedeutet nicht nur Chancen, sondern auch die Entdeckung einer vielfältigen Wirklichkeit, die zum ethnozentrischen Weltbild vieler Polen nicht passt. Autoren einer Studie zu diesem Thema weisen darauf hin, dass der Zeitraum zwischen 2002 und 2012 durch ein stabiles Verhalten der Polen den Immigranten gegenüber gekennzeichnet war (vgl. Pasamonik \& Duch-Krzystoszek, 2018, S. 5). Eine sichtbare Wende begann 2014, als polnische Bürger auf die Nachrichten über die vom ISIS inspirierten Terrorangriffe, später auch über die Migrationskrise und über die Verteilung der Migranten innerhalb der EU mit einer wachsenden negativen Haltung den 
Einwanderern gegenüber reagierten. Weil ein ähnlicher Stimmungswandel auch für andere europäische Länder symptomatisch war, wagten die Autoren der erwähnten Studie das Fazit, Polen hätten sich einem allgemeineuropäischen Verhaltensmuster angepasst, d.h. sie seien den Migranten derselben Rasse vielmehr als den Einwanderern aus anderen Regionen gegenüber offener gewesen. Dieser Platzierung Polens zwischen dem Westen und dem Osten Europas (vgl. Pasamonik \& Duch-Krzystoszek, 2018), von dem in der Studie die Rede ist, liegt aber ein polenspezifischer Faktor zugrunde: Es gibt fast keine Einwanderung aus muslimischen Ländern, darunter aus der Türkei. In diesem Fall haben wir mit einem ähnlichen Phänomen, wie in Sachsen oder Thüringen zu tun, nämlich mit einer wachsenden Feindschaft der "Islamisierung" gegenüber bei einem relativ geringen Anteil von Ausländern an der Bevölkerung beider Bundesländer. Im Jahre 2012, also noch vor der Migrationskrise wurde diese Eigenart der polnischen Xenophobie wie folgt definiert:

Die charakteristischen Merkmale des polnischen Populismus, das ihn von den Populismen in anderen europäischen Ländern unterscheidet, sind ein starker Bezug zum Katholizismus und die Institution der katholischen Kirche. Zudem zeichnet inn - aus offensichtlichen Gründen - ein Mangel an antimigrantischem Diskurs aus. (Syska, 2012, S. 30)

Die Entwicklung in den letzten Jahren brachte diesen Befund auf den Prüfstein, denn in Polen erschienen in einer bisher unbekannten Anzahl sowohl ethnisch und sprachlich ,verwandte' Ukrainer, als auch ,Fremde' aus der fernen Türkei. Die Motivationen und der Aufenthaltsdauer beider Gruppen sind unterschiedlich - einerseits zahlreiche, gut integrierte Arbeitsmigranten und Studenten aus der Ukraine, andererseits (Arbeits) migranten aus der Türkei, deren Zahl auf 4100 bis 4500 geschätzt wird (Andrejuk, 2019, S. 165) sowie türkische ERASMUS-Studenten, die sich in Polen höchstens für ein oder zwei Semester aufhalten - 1807 Studenten, darunter 504 Frauen, im Studienjahr 2017/2018 und 163 Hochschulabsolventen (Andrejuk, 2019, S. 165). Während die Meinungen der Polen über Ukrainer sehr stark (zum Teil aus historischen Gründen), polarisiert sind, wird die Abneigung gegen Türken (42\%) häufiger als Sympathie (23\%) bekundet, was einen weiteren Widerspruch darstellt: Obwohl das Verhältnis zu den Russen sehr stark historisch belastet ist, bekunden die Polen ihre Sympathie den russischen ,Erbfeinden' häufiger (31\%) als den uns seit einigen Jahrhunderten friedlich eingestellten Türken (23\%) (OmyłaRudzka, 2017). Vor dem Hintergrund der oben genannten Zahlen ist es schwer, sich des Eindrucks nicht zu erwehren, dass die zum großen Teil laizistischen Türken - anders als 
indonesische Studenten, die an polnischen Universitäten Pausen für Salāt-Gebete beantragen (Willma, 2019) - in Polen quasi stellvertretend für Migranten aus dem Nahen Osten gehalten werden und als vermeintliche Islamisten auf Abneigung stoßen.

Der Leitfaden für Hochschulen, der von der Stiftung Polnischer Rektoren 2007 verfasst wurde, bestimmt im Pkt. 16, dass Universitäten in Polen apolitische Institutionen sind und vom jeweiligen Rektor vor politischer Instrumentalisierung geschützt werden sollten (vgl. Kodeks, 2007, S. 14). Gleichzeitig werden Hochschullehrer angeregt, sich am öffentlichen Leben zu beteiligen. Dieses Recht nahm Bassam Aouil (2015 Professor am Institut für Psychologie an der Kazimierz-Wielki-Universität Bydgoszcz und Vorsitzender des Polnischen Therapeutischen Verbands) in Anspruch, um den plötzlichen Stimmungswechsel in Polen am Anfang der Migrationskrise zu diagnostizieren:

Ich bin vor 22 Jahren aus Syrien nach Bydgoszcz gekommen. Die Polen haben mir nie etwas angetan. Nach der Rückkehr aus einem dreiwöchigen Aufenthalt im Libanon war ich aber verblüfft. Es war ein Polen voller Fremdenfeindlichkeit und Hass. Was ist mit Euch passiert?. (Lewińska, 2015)

Nach Jahrzehnten der ökonomisch und politisch motivierten Auswanderung in den Westen stellte sich während der Migrationskrise heraus, dass das Interesse an Polen als Studienort steigt:

\begin{abstract}
Beispielsweise studierten nach Angaben des Statistischen Hauptamts (Główny Urząd Statystyczny - GUS) im Jahr 2016 65.793 Ausländer in Polen gegenüber 4.300 im Jahr 1990/91. Aktuell stellen sie 1,5 Prozent der Studenten an polnischen Hochschulen dar. Im Falle der Bildungsmigration wird ebenfalls die Dominanz der ukrainischen Bürger sichtbar: Sie stellen über 53 Prozent der Gesamtheit der ausländischen Studenten dar. (Segeš Frelak, 2018, S. 2)
\end{abstract}

Im Studienjahr 2016/2017 betrug die Zahl türkischer Studenten nach Angaben des Portals studyinpoland.pl (Raport, 2017) 1471 Personen (6. Platz auf der Liste). Sowohl die Präsenz der Studenten aus der Türkei in Polen als auch die Vermittlung der deutschtürkischen Literatur und Kultur im Lehrprogramm der Auslandsgermanistik stellen einen didaktischen Mehrwehrt dar: Neben der Möglichkeit, die Wirklichkeit der Bundesrepublik aus einer völlig anderen Perspektive kennenzulernen, bietet sie auch zusätzliche landeskundliche und soziale Kompetenzen - auch im Umgang mit 
Einwanderern in Polen. Die Etablierung einer deutschsprachigen Literatur der in Deutschland lebenden Polen und die Existenz einer großen polnischstämmigen Population in der BRD bedeutet zwar die Notwendigkeit, sich mit der polnischen Migrationsgeschichte auseinanderzusetzen, dennoch liefert das Schaffen deutschtürkischer Autoren mehr Anregungen für Diskussionen über universale Fragen, wie die Wahrnehmung von Orient und Okzident, Frauenrechte, religiöser Radikalismus und nicht zuletzt Integrationsprozesse, die bei Türken und Polen unterschiedlich verlaufen (vgl. Loew, 2014).

\section{Welche didaktischen Potenziale stellt die Kultur der Deutschtürken dar?}

Umut Balcı bezieht sich im Artikel über die Verwendung der Migrantenliteratur auf interkulturelle Lernprozesse im DaF-Unterricht in der Türkei. Der Autor stellt u.a. die Frage nach Kompetenzen, die die Vermittlung des deutschtürkischen Schaffens fördern kann (Balcl, 2010) und geht davon aus, dass kein Text eine festgelegte Bedeutung hat - sie wird erst im Zusammenspiel von Wortlaut und Leser hergestellt. Im Hinblick auf die Vertiefung des landeskundlichen und kulturhistorischen Wissens ist diese Vorgehensweise deshalb von größter Bedeutung, weil sie - wie Balcı dazu anmerkt - die Wechselwirkungen zwischen politischen, gesellschaftlichen und sozialen Ereignissen und ihrer Bedeutung aufdecken lässt (Balcl, 2010). Überraschend klingt aber der Befund des türkischen Germanisten, der mit seinen Studenten in der Türkei Texte der Migrantenliteratur analysierte:

Die Studierenden kannten davor die genannte Subkultur gar nicht. Die Themen wie z.B. Kulturaustausch, Integration, Assimilation, Identitätsproblematik, Heimweh, Einsamkeit etc. waren für die Studierenden ganz neu, weshalb sie sich für diese Themen sehr interessierten. (Balcl, 2010, S. 155)

Die Erkenntnis, dass die im vorigen Satz zitierten Problemfelder selbst den türkischen Studenten (die ungefähr demselben Kulturkreis wie Migranten abstammen) neu erscheinen, ist der beste Beweis dafür, dass die ethnische Herkunft oder die Sozialisation in einer bestimmten Population keine Voraussetzung für eine kompetente Beschäftigung mit dieser Literatur sein müssen.

Die Frage nach der interkulturellen Motivation im Germanistikstudium, vor allem im Fremdsprachenunterricht, wurde im Rahmen einer vergleichenden Analyse von Maciej 
Mackiewicz untersucht. Der Germanist von der Adam-Mickiewicz-Universität Posen kam zum folgenden Fazit:

Dennoch sind die Unterschiede in der Motivation der amerikanischen und der polnischen DaF-Lernenden eher groß, wobei in Polen vor allem instrumentelle Motive und in den USA neben den instrumentellen auch interkulturelle Motive ziemlich stark präsent sind. Generell spielen in Polen extrinsische Motive eine größere Rolle als in den USA. (Mackiewicz, 2016, S. 90)

Trotz der an polnischen Hochschulen sichtbaren Tendenz zur Anpassung des didaktischen Angebots an die Anforderungen des Arbeitsmarktes, ist die traditionelle literaturhistorische und kulturwissenschaftliche Komponente aus dem germanistischen Lehrprogramm kaum wegzudenken. Es bleibt nur die Frage nach der didaktischen Bedeutung der deutschtürkischen Migrantenkultur für polnische Hochschulgermanisten, die nachfolgend an einigen Beispielen besprochen wird.

Angesichts der Tatsache, dass Polen anders als Deutschland erst im letzten Jahrzehnt zu einem Einwanderungsland geworden ist und dass die (überwiegend ukrainischen) Migranten aus einem Nachbarland stammen und sich schnell integrieren, ist es auch notwendig, auf einige Unterschiede hinzuweisen, mit denen polnische Lehrkräfte und StudentInnen aufgrund der relativen ethnischen Homogenität ihres Landes in diesem Ausmaß noch nicht konfrontiert wurden. Die im Artikel von Balcı angeführten und von Berberoğlu bereits 1995 formulierten Kriterien sind, wenn auch im unterschiedlichen Ausmaß, eine neue und derzeit nur ansatzweise denkbare Aufgabe, die vor der Studienrichtung Germanistik in Polen steht:

\begin{abstract}
Interkulturelles Lernen im DaF-Unterricht realisieren zu können, ist abhängig von einigen Kriterien, die sowohl die Lehrkräfte in den Universitäten und ihre Fähigkeiten als auch die gewählten Methoden betreffen. Berberoğlu greift diese Kriterien in drei Bereichen auf; das sind: eine offene sowie kreative Haltung, die Kenntnis der diversen Kulturen, die in den Schulen repräsentiert sind und die Verfügbarkeit adäquater pädagogischer sowie didaktischer Hilfsmittel. (Balcı, 2010, S. 144)
\end{abstract}

Über Arbeitsmethoden, die sich im DaF-Unterricht mit polnischen Lernenden bewähren können, schreibt Kamila Chmielewska. Der Artikel umfasst u.a. eine detaillierte Einteilung der Unterrichtseinheit und berücksichtigt sowohl Methoden der Arbeit mit 
audiovisuellen Medien, als auch themenspezifische Aspekte (Auto- und Heterostereotypen, Multikulturalität, Recherchen zum Begriff "Gastarbeiter", Identität, Migration usw.). Ein solcher Ansatz knüpft indirekt an die vorhin zitierten Kriterien von Umut Balcı an. Die Autorin schreibt nämlich: „Um einem realitätstreuen Bild des Alltagslebens in Deutschland gerecht zu werden, sollte man auf diese kulturelle Vielfalt auch im DaF-Unterricht eingehen" (Chmielewska, 2014).

Von einem ähnlichen Standpunkt geht Anna Warakomska aus und formuliert dabei weitere didaktische Prämissen und konkrete Vorschläge (u.a. in Anlehnung auf eigene Erfahrungen):

\begin{abstract}
Darüber hinaus kann diese Literatur, wie das übrigens auch publizistische Texte zum Thema vermögen, den Lernern des Deutschen in Polen (zumindest auf universitärer Ebene) viel Wissen über die Probleme der Migration, über kulturelle Verortung, über Ähnlichkeiten und Differenzen in den Lebensweisen von Einwanderern und den BioDeutschen, also Einheimischen, vermitteln und dies in einer genauso gut verständlichen wie anmutigen Sprache. Daher eignet sich die Literatur der Migranten und ihrer Nachfahren meiner Ansicht nach nicht nur als Gegenstand germanistischer Forschung, sondern auch Lehre. Und dies insbesondere in Polen, wo die Migrantenproblematik bisher überwiegend nur eine Pressexistenz hatte. (Warakomska, 2017)
\end{abstract}

Weil die Hochschulen in Polen ihre Lern- und Lehrziele sowie deren Bewertung nach dem ECTS-System festlegen, ist es möglich, die Lernerergebnisse des jeweiligen Studiengangs und Unterrichtsinhalte autonom zu definieren, um dadurch u.a. im Bereich sozialer Kompetenzen die Offenheit der Studierenden für kulturelle Vielfalt zu fördern. Die Vermittlung der deutschtürkischen Kultur kann deshalb in unterschiedlichen Fächern realisiert werden. Dieser Themenkomplex ist ein fester Bestandteil solcher Lehrveranstaltungen, wie Landeskunde und Kulturgeschichte der D-A-CH-Länder, Kulturwissenschaft, Geschichte der deutschen Literatur und Diplomseminar (Bachelor und Master). Seit 2003 werden im Rahmen dieser Fächer am Institut für Germanistik der Kazimierz-Wielki-Universität Bydgoszcz vom Autor des vorliegenden Artikels interkulturelle Inhalte vermittelt. Darüber hinaus können Studierende der Angewandten Linguistik (Englisch/Deutsch und Deutsch/Russisch) eine monographische Vorlesung zur Multikulturalität in Deutschland als ein fakultatives Fach wählen und den Studenten der Soziologie steht die Lehrveranstaltung zum Thema Ethnizität-MultikulturalitätGrenzgebiete zur Wahl. Weil im Studiengang „Kulturwissenschaft“ das Wissen um die Kulturen der Nachbarländer, darunter die Grundlagen der deutschen Kultur, vermittelt 
werden, bietet sich die Möglichkeit, die Studierenden auf die Bedeutung der Migrantenkultur in der Kulturentwicklung Deutschlands zu sensibilisieren.

Die Einbindung der auf deutschtürkische Filme, Literaturwerke oder Comedy-Shows bezogenen Lehrinhalte kann im Laufe der Zeit dazu führen, dass die anfangs als exotische und zum Teil unverständliche Thematik von den Studenten als ein vollberechtigter Teil des Germanistikstudiums anerkannt werden kann.

\section{Polnische Studenten über die Kultur türkischer Einwanderer in der BRD}

Die folgende Analyse umfasst insgesamt 24 Bachelor- und Masterarbeiten, die vom Autor des vorliegenden Artikels am Institut für Germanistik der Bydgoszczer Universität zwischen 2007 und 2019 betreut wurden. Die von StudentInnen formulierten Themen lassen sich in 4 thematische Gruppen aufteilen: Integrationsprobleme (11 Arbeiten), Frauen(rechte) - 9, Satire - 2, Deutsche über Türken - 2 .

In der ersten Gruppe findet man Arbeiten zu den folgenden Themen: Kluft zwischen Orient und Okzident in den Texten von Necla Kelek, Konfrontation von zwei (Kultur) welten im Gegen die Wand von Fatih Akın, Kultur als Förderelement der Integration, deutsche Schulen als Orte der Integration, Das Bild der Deutschtürken im Wandel der Zeit bei Günter Wallraff und Feridun Zaimoglu, polnische und türkische Einwohner Berlins in einer vergleichenden Analyse, berufliche Biografien der Deutschtürken im Spiegel der Presse, interkultureller Dialog und seine politisch-rechtlichen Bedingungen, Bilder der Integration im Werk von Yasemin Şamdereli und Fatih Akın.

Der Themenbereich Frauen(rechte) nimmt den zweiten Platz und wurde am Beispiel von solchen Fragen, wie Ehrenmord, Zwangsheirat, Medienbilder von muslimischen Frauen in Deutschland, Bevormundung der muslimischen Frau, Leben zwischen zwei Kulturen und Identitätssuche, Kopftuchstreit, Liebesgeschichten mit Migrationshintergrund und Vater-Tochter-Konflikt besprochen. Zum Gegenstand der Forschung wurden Texte und Filme von Serap Çileli, Seyran Ateş, Alice Schwarzer, Renan Demirkan, Necla Kelek, Hatice Akyün und Feo Aladağ.

Den thematischen Schwerpunkt Satire repräsentieren in diesem Verzeichnis 2 Arbeiten über Enthowitze von Kaya Yanar (in Bezug auf Deutsche, Türken und Polen). 
Die letzte Gruppe umfasst das aktuelle Bild der Ausländer (darunter Türken) in den Filmen und Texten von Günter Wallraff und Thilo Sarrazin.

Aus dem oben präsentierten Überblick lassen sich einige Schlussfolgerungen ziehen:

1) Die Probleme der Integration dominieren in den studentischen Forschungsarbeiten. In Anbetracht der Tatsache, dass die meisten Arbeiten zu diesem Thema vor der Migrationskrise, also auch vor dem antiislamischen und fremdenfeindlichen Stimmungswechsel entstanden, kann man den Schluss ziehen, dass ihre AutorInnen eine autonome Entscheidung über die Themenwahl getroffen haben, ohne sich dabei nach politischen Denkmustern zu richten.

2) Ähnlich wie in der polnischen kultur- und literaturwissenschaftlichen Forschung zur türkischen Community in der BRD wird dieser Themenbereich definitiv von Frauen bevorzugt. Einerseits mag es daran liegen, dass philologische Studienrichtungen hauptsächlich von Frauen gewählt werden, andererseits beobachtet man in Polen eine Polarisierung des Diskurses über das Frauenbild (im Spannungsfeld zwischen Religion, Tradition und der liberalen Gegenwart), die diese Themenwahl beeinflussen konnte.

3) Das Thema Satire, besonders Fernsehsatire ist ein Teil der audiovisuellen Kultur, die zu den bevorzugten Forschungsthemen unter den Studenten gehört - nicht zuletzt als eine Alternative zur Literatur.

4) Die in der letzten Gruppe analysierten Bilder der Türken und anderer Ausländer erfordern einen komplexen Blick auf das Werk von Günter Wallraff, um den Weg des kritischen Reporters zwischen Ganz unten und Schwarz auf Weiß zu analysieren. Die Beschäftigung mit dem Buch von Thilo Sarrazin (Deutschland schafft sich ab. Wie wir unser Land aufs Spiel setzen) war eine Reaktion auf eine groß angelegte Debatte, in der die Grenzen der Meinungsfreiheit, des Tabubruchs und der Xenophobie ausgelotet wurden.

\subsection{Analyse von Bachelor- und Masterarbeiten. Befunde der Umfrage zum Thema ,Literatur und Kultur türkischer Einwanderer' im Lehrprogramm der Germanistik (Kazimierz-Wielki-Universität Bydgoszcz, Polen)}

\section{Ziel}

Um das Interesse der Studenten an der deutsch-türkischen Kultur aufzuzeigen, wurde eine Umfrage durchgeführt. 


\section{Methode der Datenerhebung und Datenauswertung}

In der Befragung werden qualitative wie quantitative Erhebungs- und Auswertungsverfahren angewendet.

\section{Vorgehensweise}

An der Befragung nahmen jeweils 34 (2007) und 13 (2020) Probanden teil. Der Zeitrahmen dieser Untersuchung (2007-2020) erstreckt sich über eine Phase, die durch ein stabiles Verhältnis zu anderen Nationalitäten gekennzeichnet war, dann über den Höhepunkt der (antimuslimisch ausgerichteten) Fremdenfeindlichkeit in den Jahren 2014-2015 (Terroranschläge und Migrationskrise) und endet mit der Konsolidierung der Macht durch die Partei Recht und Gerechtigkeit (PiS), für die u.a. eine ethnozentrische Narrative charakteristisch ist (vgl. Saryusz-Wolska et. al., 2018). Obwohl das Ergebnis einer solchen Untersuchung praktisch nur im Moment der Datenerhebung gültig ist, gibt sie dennoch bei der wiederholten Datenerhebung in der späteren Zeit Auskunft darüber, ob veränderte politische Bedingungen (wie der Rechtsruck in Polen) das Verhalten einer bestimmten Altersgruppe beeinflussen (vgl. Wolff-Powęska, 2018). Die Umfrage wurde an den Studenten des fünften (2007) und dritten Studienjahres (Master- und BachelorAbschluss) durchgeführt (in beiden Fällen vor den Seminaren über die Migrantenkultur). Die unterschiedliche Probandenzahl und die Tatsache, dass sie zwei Stufen repräsentieren, hängt damit zusammen, dass das Interesse am Germanistikstudium (und damit auch die Studentenzahl) mittlerweile kleiner geworden ist und dass die Studenten im Rahmen eines konsekutiven Studiums immer häufiger eine andere bzw. verwandte Studienrichtung wählen (z.B. angewandte Linguistik).

\section{Ergebnisse}

1. Was wissen Sie von der deutschtürkischen Literatur und Kultur?

2007: 3 Personen äußerten sich über Musik, beschränkte Frauenrechte und Größe der deutschtürkischen Community. Andere Befragten machten dazu keine Angaben.

2020: 12 Antworten. 8 Personen gaben an, dass sie davon nichts wissen. 3 Personen war der Name Fatih Akın bekannt.1 Person erwähnte den Schauspieler Erdoğan Atalay. 
2. Ist die Besprechung der deutschtürkischen Kultur und Literatur im Germanistikstudium notwendig?

2007: 8 Personen antworteten mit „Ja“, aber ohne Begründung, 21 wählten die Antwort "Ja" und begründeten sie damit, dass die Kultur der Deutschtürken ein bedeutender Teil der Kultur in Deutschland ist und deshalb während des Germanistikstudiums besprochen werden sollte. 2 Personen antworteten mit „Ja, aber im beschränkten Ausmaß“, 1 Person: „Ja, denn wir haben Angst vor Muslimen“, 1 Person: „Ja, denn ich kenne einige Türken". 1 Person machte dazu keine Angaben.

2020: 4 Antworten mit Vorbehalten: deutsche Kultur ist wichtiger, es ist nicht notwendig, aber auf jeden Fall interessant oder aufgrund einer hohen Ausländerzahl ist es ein Wissen, dass sich lohnt, man kann die Unterschiede z.B. durch Literatur aufzeigen. Ansonsten positive Antworten u.a. mit dem Hinweis darauf, dass man dadurch besser weiß, wie man sich taktvoll verhalten soll, dass die deutschtürkische und deutsche Kultur miteinander verbunden sind und dass die Größe der türkischen Community auch ein Argument dafür sein könnte.

3. Kann die deutschtürkische Perspektive in der Kultur/Literatur Ihr Deutschlandbild verändern?

2007: 12 Personen antworteten mit „Ja“, 10 mit „Nein“, darunter 2 mit „verändern nicht, sondern bitte streichen den Blick verschärfen“, 12 Personen machten keine Angaben.

2020: Antworten mit „Nein“: 1 Person mit der Begründung, dass es in Deutschland schwer sei, „einen ,echten' Deutschen zu finden. 1 Person antwortete mit „Ich weiß es nicht". In den positiven Antworten machte 1 Person die Angabe, dass man Deutschland dadurch nicht mehr als eine Einheit sieht, sondern in seiner Vielfalt. Eine andere Person stellte fest: Deutschland verändert sich durch den Multikulturalismus.

4. Glauben Sie, dass polnische Germanistikstudenten ihre Kompetenzen um die Informationen über die deutschtürkische Kultur erweitern sollen?

2007: 24 Personen antworteten mit „Ja“, 8 Personen mit „Ja, aber im beschränkten Umfang", 1 Person antwortete mit "Nein, die jetzigen Kompetenzen sind ausreichend“, 1 Person stellte die Frage „Warum nicht die deutschpolnische Kultur?" 
2020: Alle befragten Personen antworteten mit „Ja“, wobei 5 auch ihre Vorbehalte formulierten: die deutsche Kultur ist wichtiger, das Wissen um die deutschtürkische Kultur nur fakultativ. Andere Antworten: Ja - wegen der Verankerung dieser Kultur in Deutschland, auch im Bereich der Sprache, was für Germanistikstudenten von besonderer Bedeutung ist, es ist auch wegen der Öffnung auf andere Kulturen wichtig.

5. Können Sie sich vorstellen, Bücher deutschtürkischer Autoren über solche Themen, wie Frauenrechte in der muslimischen Gesellschaft, Integrationsprobleme, Tradition und Gegenwart im Leben der Deutschtürken usw. in Ihrer Freizeit zu lesen?

2007: 22 Personen antworteten mit „Ja“, (5 waren an der Frauenproblematik interessiert), 7 mit „Nein“ (aus Zeitgründen), keine Angaben bei 5 Personen.

2020: 2 Personen antworteten mit "Nein". Weitere Antworten waren positiv. 2 Personen: wichtige Themen, eine Thematik, die auch uns Polen betrifft. 2 Personen drückten ihre Vorbehalte aus: dieser Inhalt ist wenig realistisch, eine schwierige Lektüre.

6. Nennen Sie stichwortartig Ihre Assoziationen mit der türkischen Kultur, Geschichte, Politik und Religiosität.

2007: Türkische Küche, Musik (Tarkan - 2 Personen) und Handwerk (Teppiche), Verleihung des Nobel-Preises an Orhan Pamuk (2 Personen), türkischer Weg zum EUBeitritt (2 Personen). 7 Personen machten keine Angaben.

2020: Der Islam (7) als die größte, aggressive und intolerante Religion in der Türkei (1 Person: für die meisten Türken ist der Islamismus kein Begriff), Benachteiligung von Frauen, religiös motivierte Mordtaten, Türkei erkannte die Teilungen von Polen nicht an, Imperialismus und zum Teil blutiger Expansionismus in der Geschichte, Mitgliedschaft in der NATO, Fernsehserien, Nationalismus, Atatürk - Vater der Modernisierung in der Türkei, der türkische Präsident.

\section{Was assoziieren Sie mit den in Deutschland lebenden Türken?}

2007: Beschränkte Frauenrechte, kinderreiche Familien, Kopftücher, Gemüsehandel, kleine Anpassungsfähigkeit, Ghettos, deutsch-türkische Ehepaare, Unverschämtheit, häusliche Gewalt, Leben auf Kosten des deutschen Sozialstaates, Probleme mit der 
deutschen Sprache, gute Regisseure und Schauspieler, in manchen Fällen gute Ausbildung, Lebensfreude, Hass der Deutschen auf die Deutschtürken. 6 Personen: keine Assoziationen.

2020: Keine Assoziationen: 2 Personen. Weitere Antworten: Gewalt, Abgrenzung, religiöse Expansion, hohe Geburtenrate, Arbeit, Tradition - Respekt, Mangel an Toleranz, Verantwortung für Terroranschläge, türkische Küche und Schnellimbiss, Kaya Yanar, Fatih Akın, Gewalt in konservativen Familien, Dienstleistungen und Handel, gelungene Integration.

\section{Fazit}

Ein Blick auf die Ergebnisse der vorliegenden Umfrage zeigt, dass bei der ersten und zweiten Datenerhebung eine relativ hohe Zahl der Probanden keine Kenntnisse über die Kultur der Deutschtürken hatte. Im Unterschied zum Jahr 2007 wurden 2020 Namen deutschtürkischer Künstler genannt (Akın und Atalay). In der großen Mehrheit waren beide Gruppen (trotz der in einigen Fällen formulierten Vorbehalte) mit der Einbeziehung dieser Problematik ins Lehrprogramm einverstanden. 2020 waren wesentlich mehr Studenten der Meinung, dass sich dadurch ihr Deutschlandbild verändern kann, was von einer Offenheit für die ethnische Vielfalt zeugen könnte. Die große Mehrheit der Studenten war ebenfalls damit einverstanden, dass ihre Kompetenzen um die Informationen über die Kultur der Deutschtürken erweitert werden sollten. Man kann ebenfalls hoffen, dass die von den meisten Studenten in beiden Datenerhebungen deklarierte Bereitschaft, Bücher deutschtürkischer Autoren zu lesen, nicht kurzlebig war. Das Wissen um die Türkei war in beiden Jahrgängen meistens durch klischeehafte Vorstellungen geprägt. In Einzelfällen spielte auch die aktuelle Berichterstattung eine gewisse Rolle (der Nobelpreis für Orhan Pamuk, Verhandlungen der Türkei mit der EU). Es wird aber auch sichtbar, dass sich bei der zweiten Datenerhebung das Wissen um die Türkei herauskristallisierte und neben den Schlüsselbegriffen aus der türkischen Geschichte (Atatürk, Modernisierungsprozesse usw.) auch aktuelle Streitfragen, wie Laizismus, Fundamentalismus oder Frauenrechte umfasste. Eine ähnliche Schlussfolgerung trifft auch für den letzten Punkt der Umfrage $z u$, in dem die Studenten vorwiegend negative soziale oder politische Erscheinungen (Terrorismus) nannten. Dennoch erwähnten sie in der Umfrage von 2020 auch Namen von Künstlern. 


\section{Schlussfolgerungen}

Gemeinsam für die Themenwahl im Diplomseminar und für die Angaben in der Umfrage ist das Interesse der Studenten an der Problematik der Frauenrechte im Islam, der häuslichen Gewalt und der Integration der Einwanderer. Dieser Befund lässt sich nicht nur damit erklären, dass ein großer Teil der Germanistikstudierenden Frauen sind, die mit ähnlichen Problemen (wenn auch indirekt) in Polen konfrontiert werden oder aus der eigenen Erfahrung nach dem Aufenthalt in Deutschland oder auch in der Türkei (Zwyczaje, 2019) schöpfen. Nicht zu überschätzen ist aber auch der Einfluss aktueller Ereignisse und der allmählich steigenden Popularität der türkischen und (seltener) deutschtürkischen Kultur in polnischen Medien. Während das Interesse der Studenten an Lehrveranstaltungen oder Diplomseminaren mit dem Schwerpunkt 'Kultur der Deutschtürken' steigt, hält sich ihre Bereitschaft, z.B. die Regimekritik in der DDR oder Bilder des Zweiten Weltkriegs in der Kultur zu analysieren, eher in Grenzen. Die Angaben in den Umfragen sowie die Themenwahl im Diplomseminar zeigen außerdem, dass dieser Wunsch nach einer thematischen Abwechslung auch die für das Germanistikstudium festgelegten sozialen Kompetenzen, wie Offenheit für andere Kulturen, Sensibilisierung für europäische Werte oder Pflege der Identität in der globalisierten Welt fördert.

Begutachtung: Extern begutachtet.

Interessenkonflikt: Es besteht kein Interessenkonflikt.

Finanzielle Förderung: Dieser Beitrag wurde von keiner Institution finanziell unterstützt.

Peer-review: Externally peer-reviewed.

Conflict of Interest: The author has no conflict of interest to declare.

Grant Support: The author declared that this study has received no financial support.

\section{Literaturverzeichnis}

Andrejuk, K. (2019). Strategizing Integration in the Labor Market. Turkish Immigrants in Poland and the New Dimensions of South-to-North Migration. Polish Sociological Review, 2(206), 157-176.

Bömelburg, H., Rohdewald, S. \& Uffelmann, D. (2016). Polnisch-osmanische Verflechtungen in Kommunikation, materieller Kultur, Literatur und Wissenschaft. Zeitschrift für Ostmitteleuropa-Forschung, Bd. 65 Nr. 2, 159166.

Chmielewska, K. (2014). Von Angst Essen Seele auf bis Almanya - deutsch-türkische Filme im interkulturellen Fremdsprachenunterricht. GLOTTODIDACTICA, XLI/1, 127-139. 
Jonczyk, A. (2014). Tekst jako forma komunikacji międzykulturowej na przykładzie twórczości niemieckotureckiej pisarki Emine Sevgi Özdamar/Text als eine Form der interkulturellen Kommunikation am Beispiel des Schaffens der deutsch-türkischen Schriftstellerin Emine Sevgi Özdamar. In Browarny et. al. (Hrsg.), Opcja niemiecka. O problemach z tożsamościq i historiq w literaturze polskiej i niemieckiej po 1989 roku, Kraków: Wydawnictwo Universitas 1989 roku (S. 223-236).

Kunicki, W. \& Zybura, M. (Hrsg.). (2011). Germanistik in Polen. Zur Fachgeschichte einer literaturwissenschaftlichen Auslandsgermanistik - 18 Porträts. Osnabrück: fibre.

Loew, P. O. (2014). Wir Unsichtbaren. Geschichte der. Polen in Deutschland. München: C.H. Beck.

Mackiewicz, M. (2016). Interkulturelle Motivation im Vergleich: DaF in Polen und den USA, Fremdsprachen Lehren und Lernen (FLuL), 45(2), 78-92.

Nayhauss, H.-Ch. \& Kuczyński, K. (Hrsg.). (1993). Im Dialog mit der interkulturellen Germanistik. Wrocław: Wydawnictwo Uniwersytetu Wrocławskiego.

Omyła-Rudzka, M. (2017). Stosunek do innych narodów. Komunikat z badań?/Verhältnis zu anderen Nationen, 21, 1-9.

Pasamonik, B. \& Duch-Krzystoszek, D. (2018). Kultura czy gospodarka? Obawy Polaków przed imigracją i imigrantami na tle innych społeczeństw europejskich/ Kultur oder Wirtschaft? Angst der Polen vor der Einwanderung und vor Einwanderer im Vergleich mit anderen europäischen Gesellschaften. Polityka Społeczna, 11(12), 2-9.

Saryusz-Wolska, M. et. al. (2018). Verordnete Geschichte. Nationalistische Narrative in Polen. Osteuropa, 3(5), 447-464.

Segeš Frelak, J. (03.07.2018). Polen, ein Immigrationsland. POLEN-ANALYSEN, 221, 1- 6.

Syska, M. (2012). Rechtspopulismus in Polen. In Rechtspopulismus in Ostmitteleuropa. Demokratien im Umbruch? Tagungsdokumentation, Herausgegeben von der Heinrich-Böll-Stiftung Brandenburg, Potsdam, 30-36.

Szabłowski, W. (2018). Merhaba. Reportaże z tomu "Zabójca z miasta moreli" i osobisty słownik turecko-polski, Warszawa: Wydawnictwo W.A.B.

Warakomska, A. (2017). Die Literatur der Migranten und ihrer Nachfahren als Gegenstand germanistischer Forschung und Lehre am Beispiel ausgewählter Werke von deutschschreibenden Autoren türkischer Herkunft. In: Ewa Żebrowska, Magdalena Olpińska-Szkiełko, Magdalena Latkowska (Hrsg.), Beiträge zur Germanistik. Germanistische Forschung in Polen Gegenstände und Methoden. Formen und Wirkungen (S. 276291), Warszawa: Verband Polnischer Germanisten.

Wolff-Powęska, A. (2018). Trommler der Revolution. Jungkonservative und Polens Rechte. Osteuropa, 3(5), 57-76.

\section{Internetquellen}

Ayşen Kaim, A. (3.11.2015). Hürrem - turecka Marysieńka? Wokół serialu "Wspaniałe stulecie” / Hürrem - eine türkische Marysieńka? Rund um die Fernsehserie „Muhteşem Yüzyıl". In: Kultura Liberalna, Nr 356 (44/2015), , https://kulturaliberalna.pl/2015/11/03/hurrem-turecka-marysienka-wokol-serialu-wspaniale-stulecie/. (Zugriff am 06.03.2020). 
Kultur der türkischen Diaspora in Deutschland und ihre Implementierung im Germanistikstudium. Ein praxisorientierter ...

Balcı, U. (2010). Vermittlung der deutschsprachigen Literatur türkischer MigrantInnen im DaF-Unterricht in der Türkei. Çukurova Üniversitesi Eğitim Fakültesi Dergisi, 39, 142-159, https://www.researchgate.net/ publication/331225335_VERMITTLUNG_DER_DEUTSCHSPRACHIGEN_LITERATUR_TURKISCHER_ MIGRANTINNEN_IM_DAF-UNTERRICHT_IN_DER_TURKEI. (Zugriff am 06.03.2020).

Lewińska, A. (21.09.2015). Syryjczyk od 22 lat w Polsce: Skąd w was tyle nienawiści?/Ein Syrer lebt seit 22 in Polen: Woher kommt so viel Hass unter euch? In: Gazeta Wyborcza - Bydgoszcz, https://bydgoszcz.wyborcza.pl/bydg oszcz/1,48722,18844743,syryjczyk-od-22-lat-w-polsce-skad-w-was-tyle-nienawisci.html?disableRedirects= true. (Zugriff am 06.03.2020).

Mikołajczuk, M. (06.03.2014). Nie tylko Pamuk - literatura turecka znów na polskich półkach /Nicht nur Pamuk-türkische Literatur wieder auf polnischen Bücherregalen. Polskie Radio Program I, https://www.polskieradio.pl/7/173/ Artykul/1068432,Nie-tylko-Pamuk-literatura-turecka-znow-na-polskich-polkach. (Zugriff am 06.03.2020).

Szostek, A (red.) (2007). Kodeks DOBRE PRAKTYKI W SZKOŁACH https://www.krasp.org.pl/resources/upload/ dokumenty/kodeks_dobrych_praktyk.pdf,. (Zugriff am 01.09.2020)

Willma, A. (19.11.2019). Muzułmańscy studenci z UMK chcą przerw na modlitwę/Muslimische Studenten an der Nikolaus-Kopernikus-Universität wollen Gebetspausen, https://pomorska.pl/muzulmanscy-studenci-z-umkchca-przerw-na-modlitwe-centrum-kultury-muzulmanskiej-bez-przesady-moga-odrobic/ar/c1-14593199. (Zugriff am 06.03.2020).

Zwyczaje urlopowe Polaków. "Zagraniczne wakacje Polaków 2019” oraz prognoza na zimę 2020 - egzotykal Urlaubsgewohnheiten der Polen. „Sommerferien der Polen im Ausland 2019” und eine Prognose für den Winter - Exotik. Polska Izba Turystyki, https://www.pit.org.pl/media/1/002/009/2364.pdf. (15.11.2019). (Zugriff am 06.03.2020).

Raport "Studenci zagraniczni w Polsce, 2017/Bericht "Ausländische Studenten in Polen, 2017", Study in Poland (2017). http://www.studyinpoland.pl/konsorcjum/index.php?option=com_content\&view=article\&id=1451 5:raport-studenci-zagraniczi-w-polsce-2017\&catid=258:145-newsletter-2017\&ltemid=100284. (Zugriff am 06.03.2020). 